OPEN ACCESS

Edited by:

Patricia Ann Champion, University of Notre Dame,

United States

Reviewed by: Jianjun Sun,

The University of Texas at El Paso,

United States

Jessica Seeliger,

Stony Brook University, United States

*Correspondence:

Christophe Guilhot

christophe.guilhot@ipbs.fr

Catherine Astarie-Dequeker

catherine.astarie-dequeker@ipbs.fr

†These authors have contributed equally to this work

Specialty section

This article was submitted to Bacteria and Host,

a section of the journa

Frontiers in Cellular and Infection

Microbiology

Received: 08 May 2020

Accepted: 08 July 2020

Published: 14 August 2020

Citation:

Augenstreich J, Haanappel E,

Sayes $F$, Simeone $R$, Guillet $V$, Mazeres $S$, Chalut $C$, Mourey $L$,

Brosch R, Guilhot $C$ and

Astarie-Dequeker C (2020)

Phthiocerol Dimycocerosates From

Mycobacterium tuberculosis Increase

the Membrane Activity of Bacterial

Effectors and Host Receptors.

Front. Cell. Infect. Microbiol. 10:420.

doi: 10.3389/fcimb.2020.00420

\section{Phthiocerol Dimycocerosates From Mycobacterium tuberculosis Increase the Membrane Activity of Bacterial Effectors and Host Receptors}

\author{
Jacques Augenstreich ${ }^{1 \dagger}$, Evert Haanappel ${ }^{1 \dagger}$, Fadel Sayes ${ }^{2}$, Roxane Simeone ${ }^{2}$, \\ Valérie Guillet ${ }^{1}$, Serge Mazeres ${ }^{1}$, Christian Chalut ${ }^{1}$, Lionel Mourey ${ }^{1}$, Roland Brosch ${ }^{2}$, \\ Christophe Guilhot ${ }^{1 *}$ and Catherine Astarie-Dequeker ${ }^{1 *}$ \\ ${ }^{1}$ Institut de Pharmacologie et de Biologie Structurale (IPBS), Université de Toulouse, CNRS-UPS UMR 5089, Toulouse, \\ France, ${ }^{2}$ Institut Pasteur, Unit for Integrated Mycobacterial Pathogenomics, CNRS UMR3525, Paris, France
}

Mycobacterium tuberculosis (Mtb) synthesizes a variety of atypical lipids that are exposed at the cell surface and help the bacterium infect macrophages and escape elimination by the cell's immune responses. In the present study, we investigate the mechanism of action of one family of hydrophobic lipids, the phthiocerol dimycocerosates (DIM/PDIM), major lipid virulence factors. DIM are transferred from the envelope of Mtb to host membranes during infection. Using the polarity-sensitive fluorophore C-Laurdan, we visualized that DIM decrease the membrane polarity of a supported lipid bilayer put in contact with mycobacteria, even beyond the site of contact. We observed that DIM activate the complement receptor 3, a predominant receptor for phagocytosis of $M$ tb by macrophages. DIM also increased the activity of membrane-permeabilizing effectors of $M t b$, among which the virulence factor EsxA. This is consistent with previous observations that DIM help Mtb disrupt host cell membranes. Taken together, our data show that transferred DIM spread within the target membrane, modify its physical properties and increase the activity of host cell receptors and bacterial effectors, diverting in a non-specific manner host cell functions. We therefore bring new insight into the molecular mechanisms by which DIM increase Mtb's capability to escape the cell's immune responses.

Keywords: macrophages, Mycobacterium tuberculosis, phthiocerol dimycocerosates, EsxA, membranolytic activity, receptors, membranes, complement receptor 3

\section{INTRODUCTION}

The envelope lipids, phthiocerol dimycocerosates (DIM/PDIM) are among the major virulence factors of Mycobacterium tuberculosis (Mtb). Apart from their structural role as components of the mycobacterial envelope, DIM are harnessed by the bacteria to manipulate host immune functions, especially during the early steps of infection, when $M t b$ encounters macrophages (Rousseau et al., 2004; Astarie-Dequeker et al., 2009; Cambier et al., 2014; Passemar et al., 2014; Barczak et al., 2017; Quigley et al., 2017). However, there is still a great deal of uncertainty about the molecular mechanisms of DIM-mediated effects in host cells. 
Our group has reported that DIM are transferred from the mycobacterial envelope to the membranes of host macrophages, and that this transfer increases the phagocytosis of bacteria (Augenstreich et al., 2019). Further investigations indicated that once inserted in the host membrane, DIM adopt a conical shape which disturbs the organization of membrane lipids (Augenstreich et al., 2019). We hypothesize that this has functional consequences for the activity of membrane-associated contributors to phagocytosis. Phagocytosis occurs through the recognition of Pathogen-Associated Molecular Patterns (PAMPs) present at the bacterial surface by receptors expressed in the host cell plasma membrane. In the case of $M t b$, phagocytosis involves multiple receptors, including the complement receptor 3 (CR3, also named CD11b/CD18 or Mac-1) (Schlesinger, 1993; Stokes et al., 1993; Cywes et al., 1996). Interestingly, blocking CR3 with antibodies significantly reduces the entry of a DIM-proficient $M t b$ strain into macrophages without affecting the uptake of a DIM-deficient mutant (AstarieDequeker et al., 2009), suggesting that the disorganizing effects of DIM on host cell membranes could target the activity of CR3.

DIM also cause membrane damage, such as phagosomal rupture and cell death (Augenstreich et al., 2017; Quigley et al., 2017). Previous work has shown that DIM act in concert with the type VII secretion system ESX-1 (T7SS/ESX-1), in a manner that involves the $6-\mathrm{kDa}$ early secreted antigenic target (ESAT-6, also known as EsxA) (Augenstreich et al., 2017; Barczak et al., 2017). EsxA has been predicted to insert into membranes and to lyse artificial and biological membranes (Hsu et al., 2003; Gao et al., 2004; De Jonge et al., 2007; Smith et al., 2008; Ma et al., 2015; Peng et al., 2016). Interestingly, the membranolytic activity of EsxA was found to depend on the lipid composition and on membrane fluidity (Augenstreich et al., 2017; Ray et al., 2019). The mechanism by which DIM and EsxA collaborate remains unclear. Barczak and colleagues proposed that the secretion of EsxA requires DIM (Barczak et al., 2017). However, previous findings indicated that the DIM-deficient mycobacterial strains, which are affected in their membranolytic activity, secrete similar amounts of EsxA as the parental strains (Augenstreich et al., 2017; Quigley et al., 2017). Recently, our group reported that the incorporation of DIM in the membrane of model liposomes enhanced EsxA activity (Augenstreich et al., 2017). Nevertheless, the origin of the membrane-disrupting activity of DIM remains obscure. Indeed, their potentiating effect on EsxA's activity is independent of their ability to rigidify membranes and is limited to membranes containing lipids extracted from macrophages (Augenstreich et al., 2017). Moreover, the membranolytic activity of EsxA itself was called into question by Conrad and colleagues who showed that the hemolytic activity of recombinant EsxA obtained from the same source was due to residual detergent used in the purification protocol (Conrad et al., 2017). Thus, the capacity of $M t b$ to permeabilize host membranes is more complex than initially thought.

It therefore remains of importance to determine how DIM contribute to the phagocytosis of $M t b$ and to the membranolytic activity of bacteria at the molecular level.

\section{MATERIALS AND METHODS}

\section{Antibodies and Reagents}

The 2LPM19c mouse antibody directed against the CD11b subunit of human CR3 (IgG1, dilution 1:20) and normal mouse IgG1 were purchased from Santa Cruz Biotechnology. The CBRM1/5 mouse antibody raised against an activation-specific epitope on the CD11b subunit of human CR3 (IgG1, dilution 1:50) was from eBiosciences. POPC (1-palmitoyl-2-oleoyl-snglycero-3-phosphocholine) was from Avanti Polar Lipids. THP-1 lipids were extracted from THP-1 cells as described previously (Augenstreich et al., 2017). C-Laurdan was synthesized as described in Mazeres et al. (2014). DIM (average $m_{w}=1,432$ $\mathrm{g} / \mathrm{mol}$ ) were purified from Mycobacterium canetti (Augenstreich et al., 2019). Stock solutions of $40 \mathrm{mg} / \mathrm{mL}$ were prepared by dissolving the dried lipids in chloroform: methanol (2:1, $\mathrm{vol} / \mathrm{vol}$ ) (Augenstreich et al., 2019). For cell treatment, DIM were dispersed in culture medium at a final concentration of $0.1 \mathrm{mg} / \mathrm{mL}$ corresponding to $70 \mu \mathrm{M}$. The other reagents were purchased from Sigma-Aldrich.

\section{Bacterial Strains and Growth Conditions}

The unmarked mas mutant (H37Rv $\triangle$ mas), the ppsE mutant $(\mathrm{H} 37 \mathrm{Rv} \triangle p p s E)$, the esxA mutant $(\mathrm{H} 37 \mathrm{Rv} \triangle e s x A)$ of $M t b$ and the mas mutant (BCG $\triangle$ mas), the spontaneous fad26 mutant (BCG $\triangle \mathrm{fad} 26$ ), the $p k s 15 / 1$ mutant (BCG $\triangle p k s 15 / 1)$ of Mycobacterium bovis BCG were characterized in Figure S1a and in previous work (Constant et al., 2002; Astarie-Dequeker et al., 2009; Simeone et al., 2010; Augenstreich et al., 2017). The recombinant BCG strains complemented with the ESX-1 system in DIM-proficient or DIM-deficient strains (BCG::ESX-1 and BCG $\triangle$ mas::ESX-1) were constructed as previously mentioned (Augenstreich et al., 2017). Bacteria were rendered fluorescent by transferring the plasmid pMV361H $g f p$ (Astarie-Dequeker et al., 2009) or the plasmid pMVmCherry derived from a plasmid pMV361eH harboring the mCherry encoding gene (Burbaud et al., 2016). All strains were cultured at $37^{\circ} \mathrm{C}$ as previously described (Astarie-Dequeker et al., 2009; Augenstreich et al., 2017).

\section{Phagocyte Culture}

The human promonocytic cell line THP-1 (ECACC 88081201; Salisbury, UK) was cultured and differentiated in macrophages with PMA, as mentioned in Augenstreich et al. (2019). Human blood purchased from the Etablissement Français du Sang (Toulouse, France) was collected from fully-anonymous nontuberculous donors. Peripheral blood mononuclear cells and human monocyte-derived macrophages (hMDM) were prepared as previously described (Astarie-Dequeker et al., 2009).

\section{Phagocytosis Assay}

Single bacteria suspensions were prepared from exponentially growing strains as previously described (Astarie-Dequeker et al., 2009; Tabouret et al., 2010). The concentration of bacteria was estimated from the optical density at $600 \mathrm{~nm}\left(\mathrm{OD}_{600}=0.1\right.$ for 2 $\times 10^{7}$ bacilli/mL). hMDM cultured in RPMI on glass coverslips were incubated at $37^{\circ} \mathrm{C}$ for $1 \mathrm{~h}$ with GFP-expressing bacteria at a multiplicity-of-infection (MOI) of 10:1 or with zymosan at MOI 
30:1. Phagocytosis was assessed by fluorescence microscopy as previously reported (Astarie-Dequeker et al., 2009; Augenstreich et al., 2019).

\section{Expression and Purification of Recombinant EsxA}

Recombinant Mtb EsxA (rEsxA) was prepared according to the step-by-step protocol provided by BEI Resources (available at csu-cvmbs.colostate.edu/Documents/dobos-rp004.pdf). The Cterminally His-tagged protein was expressed in Escherichia coli BL21(DE3)pLysS using the plasmid pMRLB.7 containing Mtb esxA (NR-50170, BEI Resources). rEsxA was then purified according to the BEI protocol, adapted by omitting the washing step with ASB-14. The protein was checked for purity by SDS/PAGE followed by Coomassie staining, and dialyzed using 3,500-MWCO dialysis tubing in $10 \mathrm{mM}$ ammonium bicarbonate, lyophilized and stored at $-20^{\circ} \mathrm{C}$. For the calcein leakage experiments, a small amount of rEsxA was weighed and solubilized in PBS at a final concentration of $2 \mathrm{mg} / \mathrm{mL}$.

\section{Assay for Measurement of Membrane Polarity}

We characterized membrane polarity using the membrane dye C-Laurdan, whose fluorescence spectrum shifts toward lower wavelengths when the membrane becomes more apolar (Mazeres et al., 2017). A supported lipid membrane of phospholipid POPC labeled with $1 \%$ C-Laurdan was formed on a $\varnothing 25 \mathrm{~mm}$ microscope coverslip using the method of vesicle fusion (Mascalchi et al., 2012). The membrane was put in contact with a suspension of $2 \times 10^{6}$ bacteria, centrifuged at $1,500 \mathrm{~g}$ for $5 \mathrm{~min}$ at room temperature to sediment the bacteria onto the membrane, and further incubated for $20 \mathrm{~min}$ at room temperature. We took two-photon microscopy images on a Zeiss LSM710 inverted confocal microscope using a $40 \times$ water immersion objective. The membrane polarity was characterized by the Generalized Polarization (GP), a ratiometric measure given by GP $=\left(I_{440^{-}}\right.$ $\left.I_{490}\right) /\left(I_{440}+I_{490}\right)$ where $I_{440}$ and $I_{490}$ are the fluorescence intensities of C-Laurdan at 440 and $490 \mathrm{~nm}$, respectively.

\section{Calcein Leakage Assays}

The membranolytic activity of compounds was evaluated on liposomes using a calcein leakage assay, as previously described (Augenstreich et al., 2017). The liposomes were composed of POPC or of THP-1 lipids supplemented or not with $10 \%$ $(\mathrm{mol} / \mathrm{mol})$ of DIM and contained a solution of $50 \mathrm{mM}$ calcein.

\section{Contact Dependent Hemolytic Activity of Mycobacterium spp}

Lytic activity of Mycobacterium spp was detected by a hemolysis assay. Exponentially-growing mycobacteria in 7H9-ADC-Tween $800.05 \%$ were pelleted by centrifugation and washed twice in PBS (Smith et al., 2008; Conrad et al., 2017). Fresh blood was centrifuged and the pellet was resuspended in isotonic $\mathrm{NaCl}$ solution and washed twice with PBS. The final red blood cells (RBC) suspension containing $1 \times 10^{7}$ cells was mixed with bacteria at MOI of 50:1, centrifuged and incubated at $37^{\circ} \mathrm{C}$ for $48 \mathrm{~h}$. The pellet was resuspended, centrifuged and the supernatant was recovered. The absorbance at $415 \mathrm{~nm}$ $\left(\mathrm{A}_{415}\right)$ was measured on a CLARIOstar 96-well microplate reader. The supernatants of cells treated with PBS or lysed with $0.1 \%$ Triton $\mathrm{X}-100$ were used as reference values for 0 and $100 \%$ hemolysis, respectively. The percentage of hemolysis was calculated using the formula: $\%$ hemolysis $=\left(\mathrm{A}_{415}\right.$,sample ${ }^{-}$ $\left.\mathrm{A}_{415, \mathrm{PBS}}\right) /\left(\mathrm{A}_{415, \text { TritonX100-A }}\right.$ 415,PBS $)$.

\section{Statistics}

All results are expressed as mean \pm standard error of the mean (SEM) for the indicated number of experiments $(n)$. Statistical analyses were performed using GraphPad Prism 6.0 (GraphPad Software Inc.) and presented in figure legends. Differences were considered significant if $p<0.05$.

\section{RESULTS}

\section{DIM Contribute to the Membrane Polarity Decrease in a Lipid Bilayer Beyond the Site of Interaction With Mycobacteria}

We previously reported that DIM are transferred from $M$. bovis BCG to the macrophage (Augenstreich et al., 2019) and decrease its overall membrane polarity (Astarie-Dequeker et al., 2009). We investigated whether this decrease is restricted to the site of contact between the bacilli and the host membrane, or extends beyond it, as the pleiotropic effects of DIM suggest. A supported POPC membrane labeled with the polarity-sensitive fluorophore C-Laurdan was put in contact with mCherry-expressing bacilli, either wild-type $M$. bovis BCG (BCG WT) or a DIM-deficient isogenic $M$. bovis BCG mutant, BCG $\Delta$ mas, with a deleted mas, encoding an enzyme essential for DIM synthesis (Azad et al., 1996). POPC is a physiologically representative lipid forming fluid membranes at room temperature. Using mCherry fluorescence and transmission images, we first searched for bacteria immobilized on the membrane (Figure 1A, left). The majority of bacteria remained in suspension and diffused in the buffer (blurred spots in Figure 1A). By two-photon microscopy, we then imaged the C-Laurdan around immobile bacteria. Bacteria absorbed C-Laurdan from the bilayer (Figure 1A, right), implying that the C-Laurdan fluorescence spectrum at the point of contact contained contributions both from the membrane and the bacteria. We discerned no difference in C-Laurdan fluorescence intensity between the two strains (Figure S1b). We enlarged the bacterial contour, determined from the C-Laurdan fluorescence, three times by 5 pixels $(520 \mathrm{~nm})$, thus defining three concentric bands around the immobile bacterium $(\mathrm{C} 1, \mathrm{C} 2$, and C3 in Figure 1B image). From the average fluorescence spectrum in these bands, we calculated the corresponding Generalized Polarization (GP) values to characterize the membrane polarity in each band. The value of GP increases when the membrane becomes more apolar. We subtracted the intrinsic GP of the membrane, averaged over three zones outside C1, C2, and C3 and far from all bacteria, from the GP of the different bands to quantify the GP difference ( $\Delta \mathrm{GP}$ ) around bacteria. GP was increased inside the bacterial contour for both strains, reflecting the apolar nature of the mycobacterial envelope. We noticed 


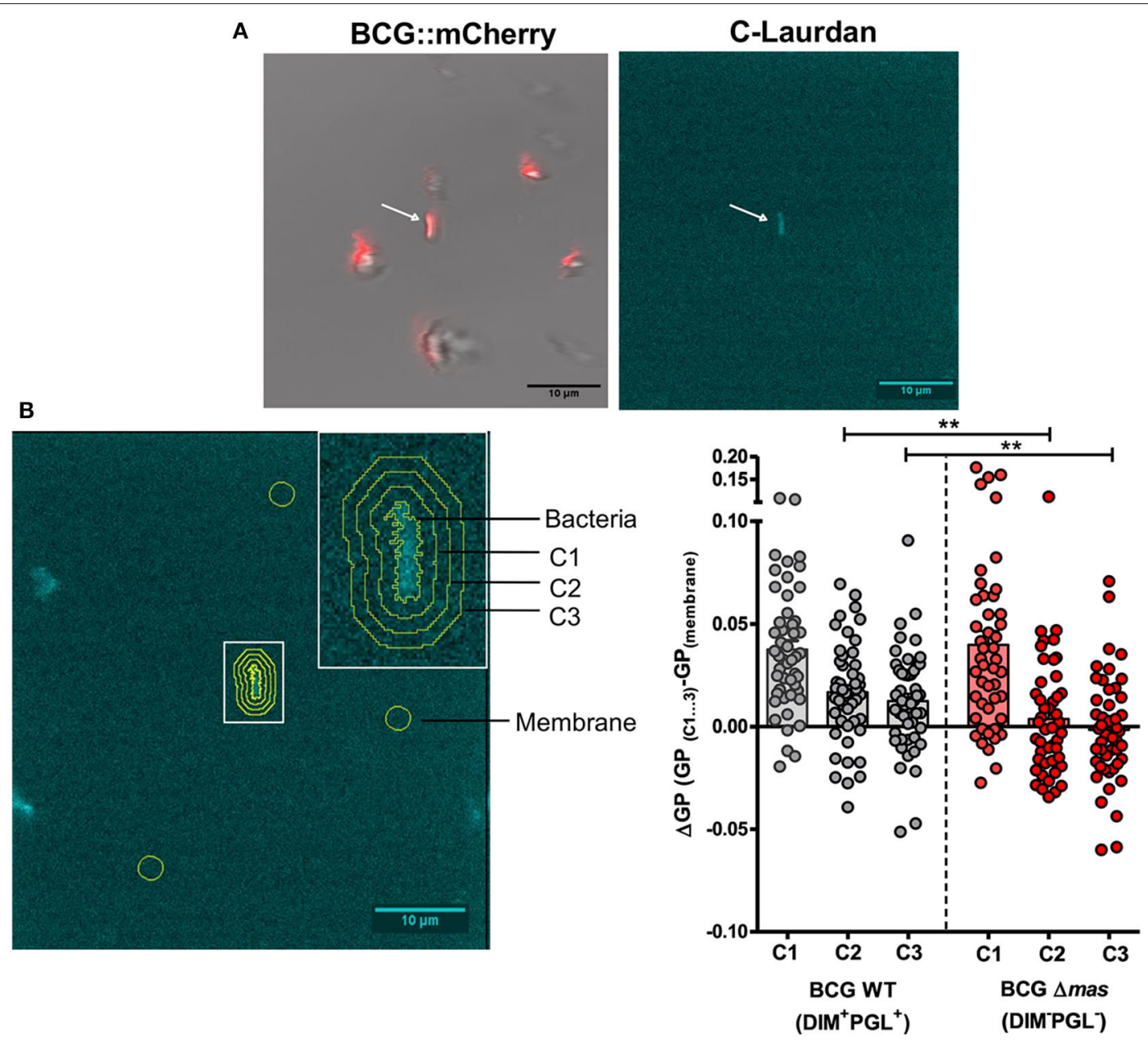

FIGURE 1 | Analysis of DIM-induced changes in membrane polarity of supported bilayers. A POPC bilayer labeled with C-Laurdan was formed on a glass coverslip and incubated with $2 \times 10^{6} \mathrm{M}$. bovis BCG::mCherry or BCG $\Delta$ mas::mCherry for $20 \mathrm{~min}$. Two-photon microscopy images were taken on an inverted confocal microscope equipped with a femto second pulsed laser (Chameleon Vision II) and with a $561 \mathrm{~nm}$ DPSS laser for mCherry fluorescence and transmission imaging. A build-in spectrometer allowed the acquisition of a fluorescence spectrum in each pixel. C-Laurdan was excited at 720 nm; its fluorescence spectrum was collected in 18 channels between 418 and $593 \mathrm{~nm}$ (channel width $9.7 \mathrm{~nm}$ ), resulting in a $\lambda$-stack of 18 images. The acquisition time per image was $31 \mathrm{~s}$ (A) Immobilized bacteria were first selected by mCherry and transmission microscopy (left panel), then the C-Laurdan spectrum was acquired around an immobile bacterium by two-photon microscopy (right panel). (B) (left panel) Contour and concentric bands around an immobile bacterium. $\Delta$ GP was calculated in these bands using the average GP of three arbitrary zones (yellow circles) selected far from C1-3 and any bacteria as the reference GP value of the membrane in each picture. (Right panel) Individual and average $\triangle G P$ values for BCG and BCG $\Delta$ mas. Each symbol in the vertical scatter plots represents the $\Delta$ GP for one bacterium. Vertical bars represent the mean \pm SEM of 47 or 49 bacteria from four independent experiments. The statistical significance of difference in the $\Delta$ GP between strains was determined using Mann-Whitney's test; ${ }^{* *} p<0.01$.

a small and similar increase in GP in zone $\mathrm{C} 1$ with respect to the membrane for both strains ( $\triangle \mathrm{GP}$ above 0 ) (Figure 1B). However, further away from the bacteria in band C2 and C3, $\triangle \mathrm{GP}$ remained positive for BCG WT, whereas GP returned to the membrane reference value for BCG $\Delta$ mas (Figure 1B). Hence, the mean GP increase was both higher and observed over a larger distance for the DIM-producing BCG WT strain than for the DIM-deficient BCG $\Delta$ mas mutant (Figure 1B). The membrane was therefore more apolar around BCG WT than around BCG $\Delta$ mas. Given the resolution of the microscope (around $250 \mathrm{~nm}$ ), the observed GP increase in C1 might be caused by bacterial fluorescence extending into $\mathrm{C} 1$. Importantly, this increase persisted up to zone C3 for BCG WT corresponding to $1-1.5 \mu \mathrm{m}$ from the edge of the bacteria (Figure 1B). This distance is too big to be caused by an optical resolution effect. In $M$. bovis BCG, the deletion of mas induces not only DIM deficiency but also the loss of the structurally similar phenolic glycolipids (PGL). We therefore made a smaller-scale test with BCG mutants deficient for DIM or PGL only. BCG $\Delta f a d 26$ mutant with a spontaneous mutation in the fad26 gene involved in DIM, but not PGL, biosynthesis (Simeone et al., 2010), displayed a lower mean $\triangle \mathrm{GP}$ than the WT BCG strain (Figure S1c). In contrast, BCG $\Delta p k s 15 / 1$ harboring a mutation which blocks PGL synthesis without modifying DIM production (Constant et al., 2002), 

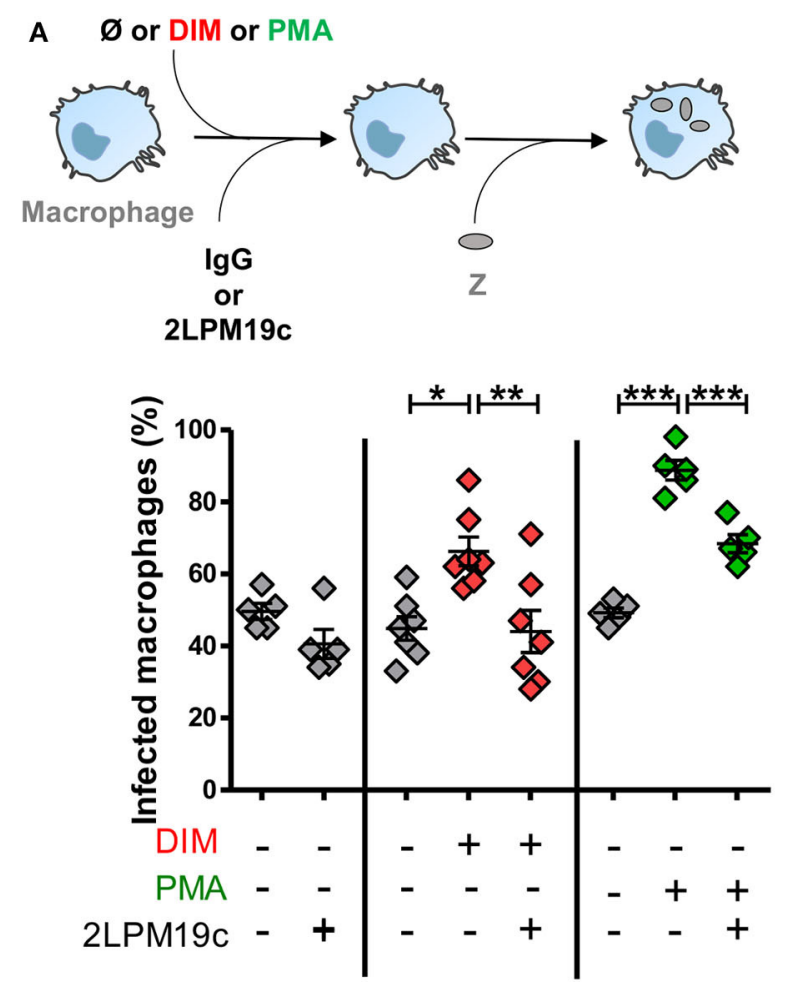
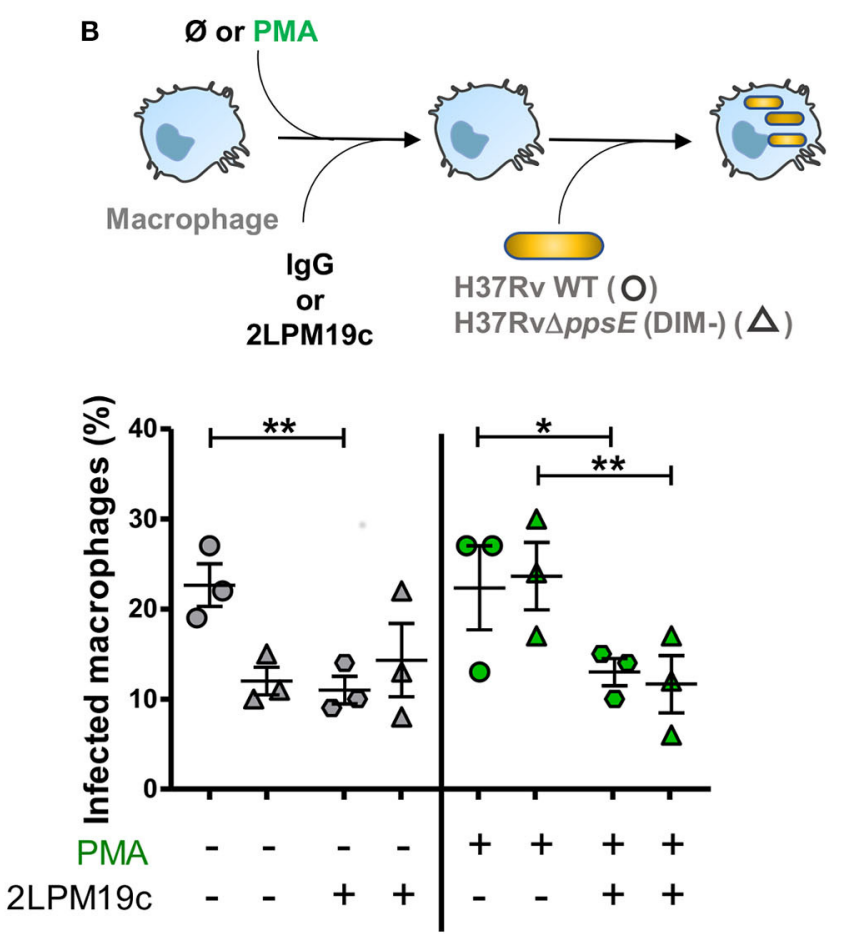

FIGURE 2 | DIM and PMA trigger the entry of the DIM-deficient H37Rv mutant and of zymosan into macrophages through a CR3-dependent process. (A) Macrophages were either left untreated (gray square) or treated at $37^{\circ} \mathrm{C}$ for $1 \mathrm{~h}$ with $70 \mu \mathrm{M} \mathrm{DIM}$ (red square) or the corresponding volume of chloroform:methanol $(\varnothing$ vehicle control, gray square) or for 15 min with 50 nM PMA (green square) or the corresponding volume of DMSO ( $\varnothing$ vehicle control, gray square). Cells were then incubated for a further $30 \mathrm{~min}$ with either the non-relevant lgG1 or $10 \mu \mathrm{g} / \mathrm{mL}$ anti-CR3 blocking antibody 2LPM19c and put in contact for $1 \mathrm{~h}$ with zymosan at MOI 30:1. (B) Macrophages were successively incubated with $50 \mathrm{nM}$ PMA (green symbol) or the corresponding volume of DMSO ( $\varnothing$ vehicle control, gray symbol) and with IgG1 or $10 \mu \mathrm{g} / \mathrm{mL} 2 \mathrm{LPM19c}$ and then exposed to GFP-expressing H37Rv (circle) or H37Rv $\triangle p p s E$ (triangle) at MOI 10:1 for 1 h. (A,B) At the end of infection, cells were rinsed, fixed and processed for the quantification of infected macrophages using a Leica 43 DM-RB epifluorescence microscope. For each set of conditions, the experiments were performed in duplicate, and at least 100 cells were counted per slide. The percentage of cells having ingested at least one bacterium, or one particle, was determined. The values are mean \pm SEM of 3-7 separate experiments. The significance of difference between control and treatment was evaluated using one-way ANOVA (A) or repeated measure ANOVA (B) followed by Bonferroni's multiple comparison test; ${ }^{\star} p \leq 0.05,{ }^{* \star} p \leq 0.01,{ }^{\star \star *} p \leq 0.001$.

behaves similar to the WT BCG strain (Figure S1c). Together, our data indicate that DIM, and not PGL, contribute to the decrease in membrane polarity which extends around the site of bacterial contact over at least $1-1.5 \mu \mathrm{m}$. They also suggest that after transfer to the host membranes, DIM diffuse laterally in the membrane.

\section{DIM Induce the Activation of Complement Receptor 3 for an Optimal Invasion of Macrophages}

We then asked whether this DIM-induced membrane perturbation could affect the phagocytic activity of CR3, the main receptor for $M t b$ entry in human macrophages. To address this question, we used zymosan, a yeast-derived polysaccharide particle, as a phagocytic prey for CR3 (Le Cabec et al., 2000). Macrophages were pre-incubated with exogenously added DIM or with a corresponding volume of chloroform:methanol dispersed in culture medium. We then evaluated the effect of the CR3-specific blocking antibody 2LPM19c on the ability of macrophages to internalize zymosan (Figure 2A). In the absence of DIM, zymosan uptake was not significantly affected by adding $10 \mu \mathrm{g} / \mathrm{mL} 2 \mathrm{LPM} 19 \mathrm{c}$, indicating that the CR3-dependent phagocytosis of zymosan is poorly efficient in resting macrophages (Figure 2A). Treatment of macrophages with DIM increased zymosan uptake by around 50\% above the level without DIM (Figure 2A). We previously related this augmentation to the conical shape of DIM (Augenstreich et al., 2019). Here, we found that this DIM-induced increase was almost completely blocked by adding 2LPM19c (Figure 2A), supporting the proposal that DIM promote the engagement of CR3 in the phagocytosis of zymosan.

We then examined whether DIM affect surface expression of CR3. Using flow cytometry, we found no difference in the expression of CD11b subunit of CR3 between untreated macrophages and macrophages treated with DIM (Figure S2a). The functionality of CR3 also depends on the activation state of the receptor (Wright and Silverstein, 1982). We showed that treating the macrophages with $50 \mathrm{nM}$ phorbol myristate acetate (PMA), a known agonist activating CR3 (Diamond and 
Springer, 1993), increased phagocytosis of zymosan compared to control cells (Figure 2A). This effect is reduced by roughly $50 \%$ in the presence of 2LPM19c. The effect of PMA on phagocytosis therefore resembles that of purified DIM, supporting the hypothesis that DIM activate CR3 and facilitate zymosan phagocytosis.

Finally, we looked at what happens in the context of $M t b$. Treatment of cells with 2LPM19c decreased by around 50\% the internalization of $\mathrm{H} 37 \mathrm{Rv} \mathrm{WT}$, confirming that $M t b$ uses mainly CR3 to invade human macrophages (Astarie-Dequeker et al., 2009). Interestingly, adding PMA did not improve the uptake of $\mathrm{H} 37 \mathrm{Rv} \mathrm{WT}$, suggesting that CR3 is already activated by $\mathrm{H} 37 \mathrm{Rv}$ (Figure 2B). In comparison, internalization of the DIM-deficient mutant $\mathrm{H} 37 \mathrm{Rv} \triangle p p s E$, which lacks a gene essential for DIM biosynthesis, is dramatically decreased and insensitive to CR3-blocking treatment, as previously described (AstarieDequeker et al., 2009). Adding PMA restored both the infectivity of the mutant and its inhibition by 2LPM19c to a level almost identical to that of the H37Rv WT strain (Figure 2B). Moreover, the CBRM1/5 antibody directed against the activation epitope of CR3 tended to block the uptake of both $\mathrm{H} 37 \mathrm{Rv}$ in untreated and PMA-treated cells and of H37Rv $\triangle p p s E$ in PMA-treated cells only (Figure S2b). Together, these data led us to conclude that DIM activate CR3, thereby ensuring an optimal invasion of macrophages.

\section{DIM Act in Concert With Mycobacterial Effectors to Promote Lysis of Biological Membranes}

We next enquired how DIM contribute to the membranedisrupting effect of $M t b$. Using our assay based on calcein leakage from large unilamellar vesicles (LUV) and mass spectrometry, we corroborated that the lytic activity of the batch of rEsxA from BEI, which we previously attributed to EsxA (Augenstreich et al., 2017), was in fact due to residual detergent ASB14 (Figure S3a-d) (Conrad et al., 2017). These observations prompted us to test a native version of EsxA (nEsxA) purified from a culture of $M t b$ (De Jonge et al., 2007). As expected, we observed $\mathrm{pH}$-dependent lytic activity of nEsxA on LUV made of POPC (Figure 3A) (De Jonge et al., 2007). The activity at pH 5 was significantly decreased after digestion by proteinase $\mathrm{K}$, ruling out the presence of detergent (Figure 3A). Based on these positive results, we investigated whether DIM could modulate the activity of nEsxA. On liposomes of THP-1 lipids without DIM, the lytic activity of nEsxA at pH 5 was $<10 \%$ (Figure 3B), well below that observed on POPC liposomes (Figure 3A). This difference can be explained by the higher membrane rigidity of THP-1 liposomes compared to POPC liposomes (Augenstreich et al., 2017; Ray et al., 2019). Nevertheless, incorporating DIM in THP-1 liposomes tended to potentiate by $35 \%$ the activity of nEsxA (Figure 3B). We also tested a recombinant version of EsxA (rEsxA) (Figure 3B) produced by us in E. coli and purified using the protocol provided by BEI Resources (Conrad et al., 2017) without the ASB-14-based endotoxin removal. We noticed that rESXA permeabilized liposomes more efficiently than nEsxA. We have no rational explanation for this difference but we observed that DIM also increased the membranolytic activity of rEsxA (Figure 3B). This effect is specific to DIM, as the incorporation of the apolar triglyceride tripalmitin in THP1 liposomes had no impact on the calcein leakage induced by rESXA ( $60 \pm 30 \%$ and $53 \pm 15 \%, n=2$, in the absence and the presence of $10 \%$ tripalmitin, respectively). We also observed that incorporation of DIM into THP-1-derived liposomes increased the lytic activity of the two pore-forming proteins, melittin and perforin-1 (Figure 3B). These observations indicated that DIM enhance the activity of different membranolytic compounds.

We also tested whether DIM work with EsxA in a mycobacterial context. To this end, we studied the hemolytic activity of several knock-out or knock-in mutants of $M t b$ and M. bovis BCG expressing combinations of DIM and EsxA. The H37Rv WT strain incubated for $48 \mathrm{~h}$ with human erythrocytes exhibited hemolytic activity $(43+8 \%, n=4)$. By comparison, the H37Rv $\Delta e s x A$ and $H 37 \operatorname{Rv} \Delta$ mas mutants were significantly less hemolytic (Figure 3C), indicating that both DIM and EsxA contribute to hemolysis. These results were confirmed in the BCG background, which has a partial deletion of ESX-1 and a functional inhibition of EsxA. Indeed, when BCG was genetically complemented with the ESX-1 region from $M t b$, EsxA secretion was re-established (Augenstreich et al., 2017) and the resulting BCG::ESX-1 strain demonstrated higher hemolytic activity than the BCG WT strain and the DIM-deficient BCG::ESX-1 $\Delta$ mas mutant (Figure 3C). Most notably, in both genetic backgrounds, the DIM-deficient strains exhibited significantly lower hemolytic activity than EsxA- or ESX-1-deficient strains, showing that DIM have an impact extending beyond EsxA/ESX-1.

\section{DISCUSSION}

The notion of DIM as bacterial effectors subverting the host's immune responses has recently emerged (Rousseau et al., 2004; Astarie-Dequeker et al., 2009; Cambier et al., 2014; Passemar et al., 2014; Augenstreich et al., 2017; Barczak et al., 2017; Conrad et al., 2017; Quigley et al., 2017; Lerner et al., 2018), but the molecular mechanisms by which DIM exert their biological activity are poorly known. We have previously demonstrated that DIM are transferred from the envelope of $M t b$ to the membranes of host macrophages during infection and locally disturb their lipid organization (Astarie-Dequeker et al., 2009; Augenstreich et al., 2019). The next step was to identify the functional role of this transfer. We now establish that DIM induce a membrane perturbation, which spreads beyond the point of contact with the bacilli and improves the activity of proteins either embedded in host membranes, like CR3, or acting on these membranes, like EsxA and other membranolytic effectors.

Using C-laurdan combined with two-photon microscopy, we showed that the membrane polarity decreased at the site of interaction of BCG with a lipid membrane. This is consistent with our previous observation of a global decrease in membrane polarity in a population of macrophages infected by BCG (Astarie-Dequeker et al., 2009). As C-Laurdan allows identifying lipid domains with different packing degrees and lipid order (Owen et al., 2010), the observed decrease in 


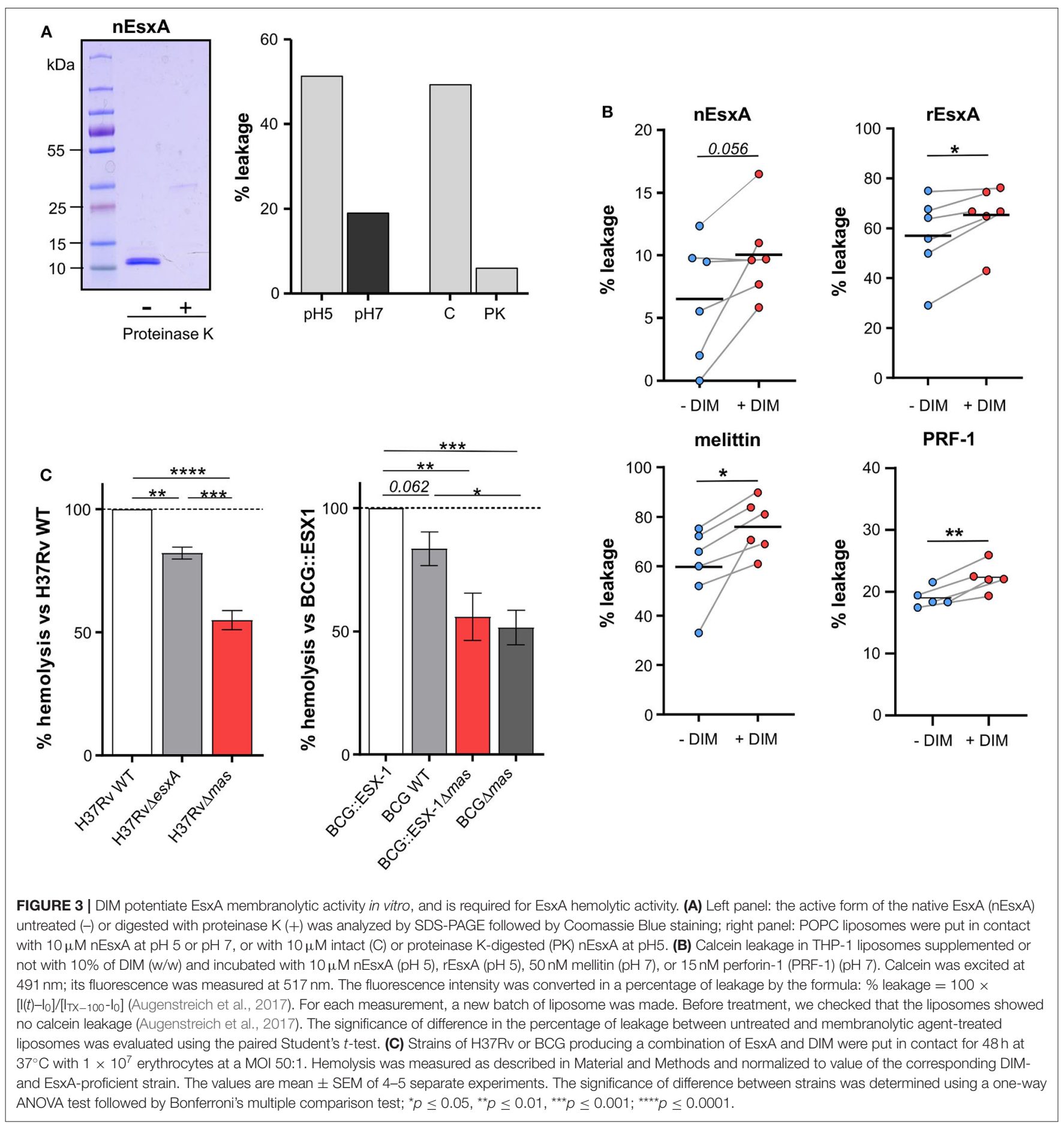

polarity strongly supports the proposal that DIM locally disturb the lipid organization of host membranes and alter their physical properties. This can also be interpreted in the light of our recent findings showing that DIM adopt a conical molecular shape in a simple phospholipid bilayer and can disorganize such a bilayer by promoting the formation of nonbilayer (inverted hexagonal) membrane phases (Augenstreich et al., 2019). Remarkably, we observed that the local decrease in membrane polarity extends over a distance of $1-1.5 \mu \mathrm{m}$ around the bacterium. These observations provide experimental support to our molecular dynamic simulations predicting diffusion of DIM inside the membrane (see (Augenstreich et al., 2019); supplementary movie). Indeed, after transfer from the bacterium to the target membranes, DIM diffuse away from the contact site, causing a gradual fading of the polarity decrease. 
Changes in membrane lipid composition and organization are generally thought to influence many cellular functions. Our findings go beyond the functional observations that $M t b$ uses envelope lipids to remodel the host membrane and divert macrophages functions (Laneelle and Tocanne, 1980; Sut et al., 1990; Welin et al., 2008; Augenstreich et al., 2019; Mishra et al., 2019). They provide evidence that insertion of lipids into host cell membranes helps the bacteria exploit membrane partners that direct the cell's responses. For instance, DIM increase CR3 activity for promoting phagocytosis. Like most integrins, CR3 is normally exposed at the cell surface in a low-activity state but can undergo changes that lead to increased activity, e.g., conformational changes leading to a state of higher affinity for their specific ligands (Liddington and Ginsberg, 2002). We found that DIM mimic at least in part the action of PMA, a known activator of CR3. For instance, DIM treatment increased the uptake of zymosan, which was almost completely abolished when CR3 was blocked by an anti-CR3 blocking antibody. Moreover, PMA restored the ability of a DIM-deficient mutant to infect macrophages equally efficiently as the WT strain, probably through the engagement of the active epitope of CR3 as assessed using CBRM1/5. All together, these data tend to indicate that DIM can also activate the CR3 receptor. Since CR3 can undergo activation yielding a conformational change that increases the affinity for its ligands, this activation would enable recognition by CR3 of PAMPs at the surface of $M t b$ leading to its uptake. Conical lipids like DIM may induce membrane curvature (Cooke and Deserno, 2006). The resulting membrane elastic stress may in turn modulate the function of integral membrane proteins (Phillips et al., 2009). It is therefore reasonable to hypothesize that DIM impose a curvature on the host's membrane that governs CR3's activation. This is consistent with the transmembrane nature of CR3 and its lateral mobility within cell membranes (Ross et al., 1992). DIM could also act on other membrane receptors cooperating with CR3 to carry out entry of mycobacteria, like CD14 which has been shown to induce an inside-out signaling pathway involving TLR2, leading to a CR3-dependent phagocytosis of M. bovis BCG (Sendide et al., 2005).

Our findings reinforce the proposal that DIM can work with MtbEsxA (Augenstreich et al., 2017; Barczak et al., 2017). Using liposomes formed of THP-1 lipids, we demonstrate that DIM enhance the activity of $M t b$ EsxA. Combined with our previous data in macrophages, these results provide a reasonable explanation for DIM's involvement in the deterioration of the phagosomal membrane by Mtb (Augenstreich et al., 2017; Quigley et al., 2017). Interestingly, this potentiating effect of DIM is not specific to EsxA but also observed for the two pore-forming proteins melittin and perforin-1, and even for the detergent ASB14 (Figure S3e). This strengthens the evidence that DIM act by perturbing their target membrane to promote the membranedisruptive activities of these molecules. Furthermore, using genedeleted $M t b$ mutants, we observed that the loss of DIM results in a lower hemolytic activity of $M t b$ than the loss of EsxA. As DIM alone have no lytic activity, this implies that additional bacterial factors are involved in this process.

In conclusion, our findings reveal molecular mechanisms by which $M t b$ exploit DIM to rewire the host cells. Given their overall hydrophobic properties, DIM are able to reshape locally the host cell membrane in a way that targets cell signaling pathways and bacterial effectors, thus supporting their multifaceted role in the virulence of $M t b$.

\section{DATA AVAILABILITY STATEMENT}

All datasets presented in this study are included in the article/Supplementary Material.

\section{AUTHOR CONTRIBUTIONS}

JA did part of the experiments, analyzed data, contributed to prepare figures, and reviewed the manuscript. EH contributed to experiments, critically analyzed the data, supervised part of the work, and reviewed the manuscript. FS, RS, and RB contributed selected $\mathrm{rBCG}$ strains and ESX-1 reagents and reviewed the manuscript. VG contributed to rESAT-6 purification. LM critically analyzed the data of rESAT- 6 purification and reviewed the manuscript. SM critically analyzed the measurement of membrane polarity and help to prepare Figure 1. CC contributed to the construction of fluorescent recombinant mCherry BCG strains. CG supervised the work and reviewed the manuscript. CA-D did part of the experiments, supervised all the aspects of the study, including execution of the experiments and analysis, wrote the manuscript, and prepared figures. All authors contributed to the article and approved the submitted version.

\section{FUNDING}

This work was supported by the European Union's Horizon 2020 Research and Innovation Program (TBVAC2020, 643381), the Agence Nationale de la Recherche (ANR-10-LABX-62IBEID, ANR-14-JAMR-001-02, and ANR-16-CE15-0003), the Fondation pour la Recherche Médicale FRM (DEQ20130326471 and DEQ20160334879), and the Center National de la Recherche Scientifique (CNRS). JA was a recipient of a Ph.D. scholarship from the French government.

\section{ACKNOWLEDGMENTS}

We thank the TRI-Genotoul Imaging facility (Toulouse, France). CA-D thanks T. Pennetier for technical assistance. We thank S. Dauvillier, E. Joly, and L. Salomé for helpful support and fruitful discussions.

\section{SUPPLEMENTARY MATERIAL}

The Supplementary Material for this article can be found online at: https://www.frontiersin.org/articles/10.3389/fcimb. 2020.00420/full\#supplementary-material 


\section{REFERENCES}

Astarie-Dequeker, C., Le Guyader, L., Malaga, W., Seaphanh, F. K., Chalut, C., Lopez, A., et al. (2009). Phthiocerol dimycocerosates of $M$. tuberculosis participate in macrophage invasion by inducing changes in the organization of plasma membrane lipids. PLoS Pathog. 5:e1000289. doi: 10.1371/journal.ppat.1000289

Augenstreich, J., Arbues, A., Simeone, R., Haanappel, E., Wegener, A., Sayes, F., et al. (2017). ESX-1 and phthiocerol dimycocerosates of Mycobacterium tuberculosis act in concert to cause phagosomal rupture and host cell apoptosis. Cell. Microbiol. 19:e12726. doi: 10.1111/cmi.12726

Augenstreich, J., Haanappel, E., Ferre, G., Czaplicki, G., Jolibois, F., Destainville, N., et al. (2019). The conical shape of DIM lipids promotes Mycobacterium tuberculosis infection of macrophages. Proc. Natl. Acad. Sci. U.S.A. 116, 25649-25658. doi: 10.1073/pnas.1910368116

Azad, A. K., Sirakova, T. D., Rogers, L. M., and Kolattukudy, P. E. (1996). Targeted replacement of the mycocerosic acid synthase gene in Mycobacterium bovis BCG produces a mutant that lacks mycosides. Proc. Natl. Acad. Sci. U.S.A. 93, 4787-4792. doi: 10.1073/pnas.93.10.4787

Barczak, A. K., Avraham, R., Singh, S., Luo, S. S., Zhang, W. R., Bray, M.-A., et al. (2017). Systematic, multiparametric analysis of Mycobacterium tuberculosis intracellular infection offers insight into coordinated virulence. PLoS Pathog. 13:e1006363. doi: 10.1371/journal.ppat.1006363

Burbaud, S., Laval, F., Lemassu, A., Daffe, M., Guilhot, C., and Chalut, C. (2016). Trehalose polyphleates are produced by a glycolipid biosynthetic pathway conserved across phylogenetically distant mycobacteria. Cell Chem. Biol. 23, 278-289. doi: 10.1016/j.chembiol.2015.11.013

Cambier, C. J., Takaki, K. K., Larson, R. P., Hernandez, R. E., Tobin, D. M., Urdahl, K. B., et al. (2014). Mycobacteria manipulate macrophage recruitment through coordinated use of membrane lipids. Nature 505, 218-222. doi: $10.1038 /$ nature 12799

Conrad, W. H., Osman, M. M., Shanahan, J. K., Chu, F., Takaki, K. K., Cameron, J., et al. (2017). Mycobacterial ESX-1 secretion system mediates host cell lysis through bacterium contact-dependent gross membrane disruptions. Proc. Natl. Acad. Sci. U.S.A. 114, 1371-1376. doi: 10.1073/pnas.1620133114

Constant, P., Perez, E., Malaga, W., Laneelle, M.-A., Saurel, O., Daffe, M., et al. (2002). Role of the pks15/1 gene in the biosynthesis of phenolglycolipids in the Mycobacterium tuberculosis complex. Evidence that all strains synthesize glycosylated p-hydroxybenzoic methyl esters and that strains devoid of phenolglycolipids harbor a frameshift mutation in the pks15/1 gene. J. Biol. Chem. 277, 38148-38158. doi: 10.1074/jbc.M206538200

Cooke, I. R., and Deserno, M. (2006). Coupling between lipid shape and membrane curvature. Biophys. J. 91, 487-495. doi: 10.1529/biophysj.105.078683

Cywes, C., Godenir, N. L., Hoppe, H. C., Scholle, R. R., Steyn, L. M., Kirsch, R. E., et al. (1996). Nonopsonic binding of Mycobacterium tuberculosis to human complement receptor type 3 expressed in Chinese hamster ovary cells. Infect. Immun. 64, 5373-5383. doi: 10.1128/IAI.64.12.5373-5383.1996

De Jonge, M. I., Pehau-Arnaudet, G., Fretz, M. M., Romain, F., Bottai, D., Brodin, P., et al. (2007). ESAT-6 from Mycobacterium tuberculosis dissociates from its putative chaperone CFP-10 under acidic conditions and exhibits membranelysing activity. J. Bacteriol. 189, 6028-6034. doi: 10.1128/JB.00469-07

Diamond, M. S., and Springer, T. A. (1993). A subpopulation of Mac$1(\mathrm{Cd} 11 \mathrm{~b} / \mathrm{Cd} 18)$ molecules mediates neutrophil adhesion to Icam-1 and fibrinogen. J. Cell Biol. 120, 545-556. doi: 10.1083/jcb.120.2.545

Gao, L.-Y., Guo, S., Mclaughlin, B., Morisaki, H., Engel, J. N., and Brown, E. J. (2004). A mycobacterial virulence gene cluster extending RD1 is required for cytolysis, bacterial spreading and ESAT-6 secretion. Mol. Microbiol. 53, 1677-1693. doi: 10.1111/j.1365-2958.2004.04261.x

Hsu, T., Hingley-Wilson, S. M., Chen, B., Chen, M., Dai, A. Z., Morin, P. M., et al. (2003). The primary mechanism of attenuation of bacillus calmette-guerin is a loss of secreted lytic function required for invasion of lung interstitial tissue. Proc. Natl. Acad. Sci. U.S.A. 100, 12420-12425. doi: 10.1073/pnas. 1635213100

Laneelle, G., and Tocanne, J. F. (1980). Evidence for penetration in liposomes and in mitochondrial membranes of a fluorescent analogue of cord factor. Eur. J. Biochem. 109, 177-182. doi: 10.1111/j.1432-1033.1980.tb04782.x

Le Cabec, V., Cols, C., and Maridonneau-Parini, I. (2000). Nonopsonic phagocytosis of zymosan and Mycobacterium kansasii by CR3 (CD11b/CD18) involves distinct molecular determinants and is or is not coupled with NADPH oxidase activation. Infect. Immun. 68, 4736-4745. doi: 10.1128/IAI.68.8.4736-4745.2000

Lerner, T. R., Queval, C. J., Fearns, A., Repnik, U., Griffiths, G., and Gutierrez, M. G. (2018). Phthiocerol dimycocerosates promote access to the cytosol and intracellular burden of Mycobacterium tuberculosis in lymphatic endothelial cells. BMC Biol. 16:1. doi: 10.1186/s12915-017-0471-6

Liddington, R. C., and Ginsberg, M. H. (2002). Integrin activation takes shape. J. Cell Biol 158, 833-839. doi: 10.1083/jcb.200206011

Ma, Y., Keil, V., and Sun, J. (2015). Characterization of Mycobacterium tuberculosis EsxA membrane insertion: roles of $\mathrm{N}$ - and C-terminal flexible arms and central helix-turn-helix motif. J. Biol. Chem. 290, 7314-7322. doi: 10.1074/jbc.M114.622076

Mascalchi, P., Haanappel, E., Carayon, K., Mazeres, S., and Salome, L. (2012). Probing the influence of the particle in single particle tracking measurements of lipid diffusion. Soft Matter 8, 4462-4470. doi: 10.1039/c2sm07018a

Mazeres, S., Fereidouni, F., and Joly, E. (2017). Using spectral decomposition of the signals from laurdan-derived probes to evaluate the physical state of membranes in live cells. F1000Res. 6:763. doi: 10.12688/f1000research.11577.1

Mazeres, S., Joly, E., Lopez, A., and Tardin, C. (2014). Characterization of Mlaurdan, a versatile probe to explore order in lipid membranes. F1000Res. 3:172. doi: 10.12688/f1000research.4805.2

Mishra, M., Adhyapak, P., Dadhich, R., and Kapoor, S. (2019). Dynamic remodeling of the host cell membrane by virulent mycobacterial sulfoglycolipid-1. Sci. Rep. 9:12844. doi: 10.1038/s41598-019-49343-2

Owen, D. M., Magenau, A., Majumdar, A., and Gaus, K. (2010). Imaging membrane lipid order in whole, living vertebrate organisms. Biophys. J. 99, L7-L9. doi: 10.1016/j.bpj.2010.04.022

Passemar, C., Arbues, A., Malaga, W., Mercier, I., Moreau, F., Lepourry, L., et al. (2014). Multiple deletions in the polyketide synthase gene repertoire of Mycobacterium tuberculosis reveal functional overlap of cell envelope lipids in host-pathogen interactions. Cell. Microbiol. 16, 195-213. doi: $10.1111 / \mathrm{cmi} .12214$

Peng, X., Jiang, G., Liu, W., Zhang, Q., Qian, W., and Sun, J. (2016). Characterization of differential pore-forming activities of ESAT-6 proteins from Mycobacterium tuberculosis and Mycobacterium smegmatis. FEBS Lett. 590, 509-519. doi: 10.1002/1873-3468.12072

Phillips, R., Ursell, T., Wiggins, P., and Sens, P. (2009). Emerging roles for lipids in shaping membrane-protein function. Nature 459, 379-385. doi: 10.1038 /nature 08147

Quigley, J., Hughitt, V. K., Velikovsky, C. A., Mariuzza, R. A., El-Sayed, N. M., and Briken, V. (2017). The cell wall lipid PDIM contributes to phagosomal escape and host cell exit of Mycobacterium tuberculosis. mBio 8, e00148-17. doi: $10.1128 / \mathrm{mBio} .00148-17$

Ray, S., Vazquez Reyes, S., Xiao, C., and Sun, J. (2019). Effects of membrane lipid composition on Mycobacterium tuberculosis EsxA membrane insertion: a dual play of fluidity and charge. Tuberculosis 118:101854. doi: 10.1016/j.tube.2019.07.005

Ross, G. D., Reed, W., Dalzell, J. G., Becker, S. E., and Hogg, N. (1992). Macrophage cytoskeleton association with CR3 and CR4 regulates receptor mobility and phagocytosis of iC3b-opsonized erythrocytes. J. Leukoc. Biol. 51, 109-117. doi: $10.1002 / \mathrm{jlb} .51 .2 .109$

Rousseau, C., Winter, N., Pivert, E., Bordat, Y., Neyrolles, O., Ave, P., et al. (2004). Production of phthiocerol dimycocerosates protects Mycobacterium tuberculosis from the cidal activity of reactive nitrogen intermediates produced by macrophages and modulates the early immune response to infection. Cell. Microbiol. 6, 277-287. doi: 10.1046/j.1462-5822.2004.00368.x

Schlesinger, L. S. (1993). Macrophage phagocytosis of virulent but not attenuated strains of Mycobacterium tuberculosis is mediated by mannose receptors in addition to complement receptors. J. Immunol. 150, 2920-2930.

Sendide, K., Reiner, N. E., Lee, J. S. I., Bourgoin, S., Talal, A., and Hmama, Z. (2005). Cross-talk between CD14 and complement receptor 3 promotes phagocytosis of mycobacteria: regulation by phosphatidylinositol 3-kinase and cytohesin-1. J. Immunol. 174, 4210-4219. doi: 10.4049/jimmunol.174.7.4210

Simeone, R., Leger, M., Constant, P., Malaga, W., Marrakchi, H., Daffe, M., et al. (2010). Delineation of the roles of FadD22, FadD26 and FadD29 in the biosynthesis of phthiocerol dimycocerosates and related compounds in Mycobacterium tuberculosis. FEBS J. 277, 2715-2725. doi: $10.1111 /$ j.1742-4658.2010.07688.x 
Smith, J., Manoranjan, J., Pan, M., Bohsali, A., Xu, J., Liu, J., et al. (2008). Evidence for pore formation in host cell membranes by ESX-1-secreted ESAT-6 and its role in Mycobacterium marinum escape from the vacuole. Infect. Immun. 76, 5478-5487. doi: 10.1128/IAI.00614-08

Stokes, R. W., Haidl, I. D., Jefferies, W. A., and Speert, D. P. (1993). Mycobacteriamacrophage interactions. Macrophage phenotype determines the nonopsonic binding of Mycobacterium tuberculosis to murine macrophages. J. Immunol. 151, 7067-7076.

Sut, A., Sirugue, S., Sixou, S., Lakhdar-Ghazal, F., Tocanne, J. F., and Laneelle, G. (1990). Mycobacteria glycolipids as potential pathogenicity effectors: alteration of model and natural membranes. Biochemistry 29, 8498-8502. doi: 10.1021/bi00488a042

Tabouret, G., Astarie-Dequeker, C., Demangel, C., Malaga, W., Constant, P., Ray, A., et al. (2010). Mycobacterium leprae phenolglycolipid-1 expressed by engineered $M$. bovis BCG modulates early interaction with human phagocytes. PLoS Pathog. 6:e1001159. doi: 10.1371/journal.ppat.1001159

Welin, A., Winberg, M. E., Abdalla, H., Sarndahl, E., Rasmusson, B., Stendahl, O., et al. (2008). Incorporation of Mycobacterium tuberculosis lipoarabinomannan into macrophage membrane rafts is a prerequisite for the phagosomal maturation block. Infect. Immun. 76, 2882-2887. doi: 10.1128/IAI.01549-07

Wright, S. D., and Silverstein, S. C. (1982). Tumor-promoting phorbol esters stimulate $\mathrm{C} 3 \mathrm{~b}$ and $\mathrm{C} 3 \mathrm{~b}$ ' receptor-mediated phagocytosis in cultured human monocytes. J. Exp. Med. 156, 1149-1164. doi: 10.1084/jem.156.4.1149

Conflict of Interest: The authors declare that the research was conducted in the absence of any commercial or financial relationships that could be construed as a potential conflict of interest.

Copyright (C) 2020 Augenstreich, Haanappel, Sayes, Simeone, Guillet, Mazeres, Chalut, Mourey, Brosch, Guilhot and Astarie-Dequeker. This is an open-access article distributed under the terms of the Creative Commons Attribution License (CC $B Y)$. The use, distribution or reproduction in other forums is permitted, provided the original author(s) and the copyright owner(s) are credited and that the original publication in this journal is cited, in accordance with accepted academic practice. No use, distribution or reproduction is permitted which does not comply with these terms. 